\title{
Literatura brasileira sobre o trabalho de enfermagem fundamentada em categorias marxianas
}

\author{
Literatura brasileña sobre trabajo de enfermería fundamentada en categorías marxianas \\ Brazilian literature on nursing work founded on marxist categories

\section{Edir Nei Teixeira Mandú', Marina Peduzzi", Brígida Gimenez Carvalho"I", Ana Maria Nunes da Silva'}

\begin{abstract}
' Universidade Federal de Mato Grosso, Faculdade de Enfermagem, Departamento de Enfermagem. Cuiabá-MT, Brasil.
"Universidade de São Paulo, Escola de Enfermagem, Departamento de Orientação Profissional. São Paulo-SP, Brasil.

I'I Universidade Estadual de Londrina, Centro de Ciências da Saúde, Departamento de Saúde Coletiva. Londrina-PR, Brasil.
\end{abstract}

Submissão: 23/11/2009 Revisão: 07/11/2010 Aprovação: 20/11/2010

\section{RESUMO}

Analisa-se a produção científica sobre trabalho de enfermagem baseada em categorias marxianas, com o objetivo de conhecer suas contribuições e tendências. O estudo é uma revisão quanti-qualitativa de 97 artigos nacionais de 1981-2008, indexados na LILACS. Abordam-se os eixos: número e distribuição periódica; temáticas; abordagem conceitual e metodológica. Identificamse: ampliação da literatura em questão, sobretudo a partir dos anos 2000; abordagem crescente dos sujeitos, de suas percepções e interação social; incorporação recente do debate sobre cuidado e gerenciamento do cuidado de enfermagem; predomínio de pesquisas qualitativas e, a partir dos anos 1990, incorporação de conceitos de outras vertentes teóricas articuladas a categorias marxianas. Evidencia-se a busca de compreensão dos movimentos, das contradições e dos dilemas da enfermagem como prática social.

Descritores: Trabalho; Enfermagem; Pesquisa em enfermagem; Literatura de revisão como assunto.

\begin{abstract}
This article discusses the Brazilian scientific literature on nursing work based on Marxist categories, with the aim of investigating their contributions and tendencies. The study is a quanti-qualitative review of 97 articles published between 1981 and 2008 , indexed in the LILACS. It is discussed: number and periodical distribution; themes; methodological and conceptual approaches. It was identified: a growing body of literature on nursing especially from 2000 onwards; the growth of approaches on the subjects, their perceptions and social interaction; the incorporation recently into the debate on caring and nursing care management; the predominance of qualitative research and, from the 1990s onwards, the incorporation of concepts from other theoretical paths linked to Marxists categories. There was evidence of a search for understanding of the movements, contradictions and dilemmas in nursing as a heterogeneous social practice.
\end{abstract}

Key words: work; nursing; nursing research; review literature as topic.

\section{RESUMEN}

Se analiza la producción científica brasileña sobre trabajo de enfermería basada en categorías marxianas, con el objetivo de conocer sus contribuciones y tendencias. Es una revisión quanti-qualitativa de 97 artículos nacionales publicados entre 19812008, indexados en LILACS. Se analizan: número y distribución periódica; temática; abordagem metodológica y conceptual. Se muestra: una literatura creciente sobre el trabajo de enfermería sobretudo a partir de los años 2000; abordagem creciente de los sujetos, sus percepções e interacción social; incorporación reciente del debate sobre cuidado y gerenciamento del cuidado de enfermería; predomínio de investigaciones qualitativas y, a partir de los años 1990, incorporación de conceptos de otras vertientes teóricas articuladas la categorías marxianas. Se evidencia la búsqueda de compreensão de los movimientos, de las contradicciones y de los dilemas de la enfermería como práctica social.

Palabras clave: trabajo; enfermería; investigación en enfermería; literatura de revisión como asunto. 


\section{INTRODUÇÃO}

Analisa-se a literatura brasileira sobre o trabalho de enfermagem que se baseia nas categorias teóricas trabalho, processo de trabalho e correlatos ancorados em Marx e em outras vertentes teóricas articuladas.

Essa produção origina-se no Brasil no início dos anos 1980, a partir da obra de Maria Cecília Puntel de Almeida e colaboradores $^{(1,2)}$, constituindo uma importante contribuição de pesquisadores brasileiros à crítica social e política da enfermagem no país, enfocando-a como prática inter-relacionada às demais práticas sociais. $\mathrm{O}$ uso das categorias em questão permite compreender que esta área, mais do que uma profissão técnica e/ ou científica, situa-se na sociedade como trabalho e, portanto, como parte do conjunto de suas influências e movimentos, de forma articulada às transformações históricas do setor saúde. Mais recentemente, esses estudos abordam questões da prática de enfermagem no Sistema Único de Saúde, com certo aprofundamento da interlocução com as ciências sociais e humanas ${ }^{(1)}$.

No Brasil, a visão marxiana do trabalho é tomada como base para a construção do pensamento social em saúde, nos anos 1970/80, influindo largamente nas produções acadêmicas da área, nas práticas assistenciais, e lutas políticas por mudanças no sistema nacional de saúde, em um contexto de democratização e crescimento da participação do Estado brasileiro nas questões de saúde ${ }^{(3)}$. Esse pensamento difunde ideias sobre o caráter sociopolítico das práticas em saúde e do processo saúde-doença, propiciando a compreensão da saúde e de suas várias profissões/trabalhos em suas conexões sociais e transformações históricas ${ }^{(1)}$.

Essa absorção se fortalece através da produção de pesquisadores como Ricardo Bruno Mendes-Gonçalves, que, com base em Marx, teoriza sobre o processo de trabalho em saúde, dedicando-se à compreensão do sentido social e político das relações entre ciência, técnica e organização da atenção à saúde, o que vai permitir o entendimento de que todo trabaIho é ato processual orientado por finalidades sociais, não se restringindo à objetividade técnico-científica ${ }^{(4)}$.

Sua produção é particularmente incorporada à pesquisa em enfermagem e fundamenta a compreensão de suas peculiaridades, contradições e interfaces com as demais práticas de saúde, e sua característica de trabalho processual composto por vários elementos interligados que se articulam entre si e a contextos concretos - objetos, tecnologias, sujeitos, produtos e finalidades sociais.

Na concepção de Marx, é através do trabalho que o homem, em relação, constrói-reconstrói criativamente a vida, gerando o social, a própria condição humana e o processo histórico. A vinculação do trabalho ao processo de geração de riquezas e ao mercado acarreta desumanização e alienação, bem como o torna desfavorável à ação autônoma dos sujeitos ${ }^{(5)}$.

Processo de trabalho é interpretado como ação humana transformadora, intencional, dirigida a um fim social, que envolve tempo, energia, objetos e aplicação de meios à produção de bens úteis à vida; como ato produtivo se inter-relaciona a movimentos de produção, consumo e circulação/ distribuição de produtos em contextos concretos, produzindo resultados segundo necessidades que incluem a reprodução das relações sociais que predominam ${ }^{(4,5)}$.

Essa categoria permite compreender que o fazer da enfermagem responde a finalidades e necessidades sociais, envolvendo sujeitos, subjetividades e reiteração e criação humana, num processo voltado à apreensão e transformação de objetos de intervenção, através da criação e aplicação de tecnologias materiais e imateriais - instrumentos, saberes, relações, comportamentos -, com a efetiva participação coletiva de seus agentes, constituindo-se em ação social limitada no tempo e espaço, mobilizada pela ação humana.

O debate sobre o trabalho em saúde e enfermagem incorpora vários conceitos correlatos ao de trabalho e processo de trabalho, como divisão social e técnica do trabalho, força de trabalho, trabalho produtivo e não produtivo, práxis, alienação do trabalho, trabalho vivo e trabalho morto, dentre outros.

Mas o uso dessas categorias não é exclusivo, na medida em que a essas se somam ideias e conceitos que integram outras vertentes teóricas. Na literatura científica, duas publicações tratam da incorporação pela enfermagem das categorias em questão nos estudos da prática. A primeira, de 1999, analisa as contribuições da pós-graduação brasileira em enfermagem, de 1983-1997, à interpretação da prática em saúde e enfermagem a partir de Marx. Aponta uma leitura inicial mais estrutural e, em especial a partir da segunda metade dos anos 1990, a incorporação de uma interpretação dialética referente à objetividade-subjetividade do trabalho com base em outras vertentes teóricas ${ }^{(1)}$. A segunda, de 2005, discute como a enfermagem brasileira aborda em artigos, do período 1993-2003, a categoria específica processo de trabaIho, assinalando uma concentração dos debates no ano 2000 e nos instrumentos do trabalho do enfermeiro(6).

A despeito de o primeiro estudo evidenciar a incorporação de novas temáticas e o uso de novas referências das ciências sociais e humanas no debate acerca da enfermagem como prática social, ao incorporar o sujeito e a dimensão da subjetividade como essenciais a sua compreensão crítica, ainda se carece de estudos que resgatem e analisem peculiaridades dessa produção. Sobretudo, parece significativo recuperar o modo como a literatura em questão aproxima-se da dimensão relacional, ético-política, micropolítica e cultural presente na prática de enfermagem, envolvendo leituras micro-sociais desta.

Assim, considerando a importância teórica do debate social da prática de enfermagem e os movimentos em curso em torno da sua teorização, se dá continuidade à análise da literatura científica sobre o trabalho de enfermagem fundamentado em Marx, em artigos publicados no Brasil entre 1981 e 2008, enfocando características gerais, especificidades de seus temas de estudo, abordagens metodológicas, verificando tendências teóricas. Pretende-se, assim, atualizar a trajetória histórica dessa produção e mobilizar futuras pesquisas que considerem características e possíveis desafios encontrados.

\section{METODOLOGIA}

Realizou-se uma revisão da literatura ${ }^{(7)}$, com análise crítica, através de abordagem quanti-qualitativa, utilizando como fonte de informações a base LILACS (Literatura Latino-Americana 
de Ciências da Saúde), dado o fato de que esta congrega a maior produção e os principais periódicos nacionais da área de enfermagem latino-americana, previamente avalizada pelos pares através das revistas científicas. Partiu-se da questão: Sobre que objetos da prática os estudos brasileiros do trabalho da enfermagem baseados nas categorias marxianas trabalho e processo de trabalho recaem e como se configura a sua apropriação teórico-metodológica?

Os critérios de inclusão da literatura adotados foram: 1) artigos que discutem o trabalho de enfermagem com base nas categorias trabalho, processo de trabalho e conceitos correlatos baseados em Marx e/ou em autores que o utilizam, independentemente da centralidade, aprofundamento, e articulação com o empírico; 2) artigos publicados entre 1981-agosto de 2008 (mês de consulta às fontes de dados), considerando o ano de 1981 um marco na abordagem social e do processo de trabalho da enfermagem no Brasil, com base em Marx, a partir de estudo de Maria Cecília Puntel de Almeida e colaboradores ${ }^{(1,2)}$.

Para busca da literatura utilizou-se o comando "trabalho", como descritor e palavra do título, acrescido das palavras "enfermagem or enfermeiro or enfermeiros or enfermeira or enfermeiras" e do filtro "Brasil" para país de publicação. Essa busca gerou 361 publicações, abrangendo perspectivas teóricas diversas. Destas, foram triadas 73, utilizando-se como critérios de exclusão: 1) estudos sem especificidade temática e/ou teórica para a pesquisa; 2) textos repetidos; 3) outras publicações que não artigos. A definição desses filtros baseou-se na consulta prévia aos Descritores em Ciências da Saúde, relacionados ao tema trabalho; e em sua testagem e definição, considerando a melhor captação da literatura em termos de volume e especificidade.

Para ampliação da revisão, mantidos os critérios de inclusão e exclusão estabelecidos, realizou-se nova busca na LILACS, por autor, a partir de lista prévia (com 43 autores). Considerou-se autores conhecidos, referenciados em estudo

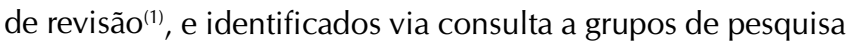
do Censo CNPq 2006 ${ }^{(8)}$. Efetuou-se, ainda, busca direta nas 15 revistas nacionais de enfermagem disponíveis no Portal de Revistas Científicas em Ciências da Saúde ${ }^{(9)}$. Por fim, verificou-se as referências bibliográficas dos estudos levantados, com o propósito de encontrar outros de interesse. Através dessas estratégias foram identificados mais 24 artigos, que somados aos anteriormente referidos totalizaram 97 publicações.

Os artigos foram lidos na integra e analisados segundo três eixos: 1) número e distribuição periódica das publicações, considerando: os anos 1980 - de emergência e desenvolvimento inicial dessa produção; os anos 1990 - de consolidação dessa produção, em um cenário de mudanças no setor saúde e na pós-graduação brasileira; e os anos 2000 - de reflexo das mudanças da década anterior; 2) os temas em debate e aspectos neles enfatizados (considerando elementos do processo de trabalho - tecnologias, força de trabalho/sujeitos e subjetividades/interações, produtos e resultados sociais, relação com o contexto -, e a sua totalidade); 3) tipo de estudo (pesquisa bibliográfica/de campo ou reflexão ou ensaio teórico), abordagem metodológica (quantitativa, qualitativa, quanti-qualitativa), e uso exclusivo de conceitos marxianos ou associados a outras correntes teóricas.
O processo analítico foi desenvolvido em duas etapas: 1) construção de banco de dados no Programa Excell, com as informações disponíveis na base consultada, acrescidas daquelas derivadas de análise qualitativa dos artigos segundo os eixos estabelecidos; 2) e análise interpretativa, mediante reconhecimento do sentido geral de cada artigo analisado e da apreensão dos sentidos específicos presentes em cada um conforme os elementos de análise estabelecidos, e processo final classificatório que resultou nas categorias empíricas apresentadas e quantificadas. Essas categorias foram consensuadas por dois dos participantes da pesquisa, tendo em vista a redução de possível viés de análise.

\section{RESULTADOS}

Entre os 97 artigos que tratam do tema trabalho de enfermagem em uma perspectiva marxiana, 2 (2,06\%) foram publicados no período 1981-1989, 21 (21,65\%) nos anos 19901999, e 74 (76,29\%) entre 2000-2008. Identificou-se um crescimento na publicação nacional de artigos sobre o tema a partir da década de 1980, concentrado sobretudo no último período e nos anos 2005, 2006 e 2007.

Os temas versam sobre: reprodução e inovações da prática de enfermagem, presente em 31 (31,96\%) artigos; espaço social de trabalho da enfermagem/do enfermeiro no setor saúde, em $22(22,68 \%)$; bases conceituais do trabalho de enfermagem, em 17 (17,53\%); processos sociais e/ou institucionais e a organização do trabalho de enfermagem, em 14 (14,43\%); recursos humanos em enfermagem, em 11 (11,34\%); e relação trabalhador de enfermagem-trabalho, em 2 (2,06\%) artigos.

Esses grandes temas ou temáticas, relativos aos elementos ou totalidade do trabalho, agregam debates com ênfases peculiares na prática de enfermagem/enfermeiro (Quadro 1), conforme apresentado a seguir.

Através do tema reprodução e inovações da prática de enfermagem, olhando para a totalidade desta, dá-se ênfase aos diferentes modos de efetivar e/ou pensar o trabalho de enfermagem/do enfermeiro, de forma articulada ao contexto de organização das práticas de saúde, de modo a evidenciar o que se reproduz e/ou vem mudando socialmente. Abordam-se contribuições, desafios e impasses da enfermagem frente a mudanças propostas e em construção no setor, considerando implicações para o saber-fazer cotidiano. Entre os estudos, alguns destacam a participação dos sujeitos na prática - trabaIhadores e usuários, seus modos de pensar e interagir.

O segundo tema mais frequente - espaço social do trabaIho de enfermagem/do enfermeiro - enfatiza: os seus elementos constitutivos; o lugar que o trabalho de enfermagem e/ou do enfermeiro ocupa no trabalho coletivo; a articulação ou dicotomia entre cuidar e gerenciar na enfermagem; a compreensão da enfermagem como vocação ou trabalho; modos de a enfermagem colocar-se frente à normatividade institucional; e a autonomia do enfermeiro no trabalho. Essas publicações abordam componentes, dimensões, articulações, dicotomias, contradições e tensões presentes no processo de trabalho da enfermagem, em sua relação com o trabalho coletivo em saúde e o espaço social ocupado por seus trabalhadores. Tal 


\begin{tabular}{|c|c|c|}
\hline Elementos articuladores da análise & Temática de Estudo & Aspecto enfatizado \\
\hline $\begin{array}{l}\text { Totalidade do trabalho - resultados } \\
\text { sociais }\end{array}$ & $\begin{array}{l}\text { Reprodução-inovações na prática } \\
\text { de enfermagem }\end{array}$ & $\begin{array}{l}\text { Modos de efetivar/pensar o trabalho de enfermagem/do } \\
\text { enfermeiro } \\
\text { Modos de efetivar/pensar o trabalho de enfermagem/do } \\
\text { enfermeiro - com destaque às inter-relações/subjetividades }\end{array}$ \\
\hline Tecnologias do trabalho (saberes) & $\begin{array}{l}\text { Bases conceituais do trabalho de } \\
\text { enfermagem }\end{array}$ & $\begin{array}{l}\text { Trabalho/processo de trabalho como fundamento na enfermagem } \\
\text { Bases orientadoras da prática assistencial e gerencial de } \\
\text { enfermagem } \\
\text { Trabalho interdisciplinar } \\
\text { Cuidado/gerenciamento do cuidado de enfermagem } \\
\text { Dimensões do processo de trabalho de enfermagem } \\
\text { Trabalho e identidade profissional do enfermeiro } \\
\text { Conhecimento produzido em enfermagem } \\
\text { Humanização do trabalho de enfermagem } \\
\text { Comunicação no trabalho de enfermagem } \\
\text { Ética /bioética no trabalho gerencial de enfermagem }\end{array}$ \\
\hline \multirow[t]{2}{*}{$\begin{array}{l}\text { Trabalho-contexto de saúde - } \\
\text { contexto social }\end{array}$} & $\begin{array}{l}\text { Espaço social de trabalho da } \\
\text { enfermagem no setor saúde }\end{array}$ & $\begin{array}{l}\text { Elementos constitutivos do trabalho de enfermagem/do } \\
\text { enfermeiro } \\
\text { Elementos constitutivos do trabalho de enfermagem/do } \\
\text { enfermeiro - com destaque às inter-relações/subjetividades } \\
\text { Trabalho de enfermagem/o enfermeiro no trabalho coletivo } \\
\text { Articulação-dicotomia entre cuidar e gerenciar na prática do } \\
\text { enfermeiro } \\
\text { Enfermagem como vocação e trabalho } \\
\text { Enfermagem frente à normatividade institucional } \\
\text { Autonomia profissional do enfermeiro }\end{array}$ \\
\hline & $\begin{array}{l}\text { Processos macrossociais e/ou } \\
\text { institucionais e a organização do } \\
\text { trabalho de enfermagem }\end{array}$ & $\begin{array}{l}\text { Mudanças contemporâneas no mundo do trabalho e implicações } \\
\text { para a enfermagem/para o cuidar/cuidado } \\
\text { Raízes sócio-históricas da prática de enfermagem/do enfermeiro } \\
\text { Conhecimento do trabalhador de enfermagem sobre } \\
\text { determinantes e condicionantes de seu trabalho } \\
\text { Divisão técnico-social do trabalho em saúde e a enfermagem } \\
\text { Condições institucionais e o trabalho de enfermagem }\end{array}$ \\
\hline \multirow[t]{2}{*}{ Trabalho-força de trabalho / sujeitos } & $\begin{array}{l}\text { Recursos humanos no trabalho } \\
\text { em enfermagem }\end{array}$ & $\begin{array}{l}\text { Formação/desenvolvimento do trabalhador e demandas da } \\
\text { prática/núcleo cuidar/cuidado em enfermagem } \\
\text { Força e mercado de trabalho } \\
\text { Regulação do trabalho de enfermagem }\end{array}$ \\
\hline & $\begin{array}{l}\text { Relação trabalhador de } \\
\text { enfermagem-trabalho }\end{array}$ & $\begin{array}{l}\text { Produção de subjetividade do trabalhador de enfermagem no } \\
\text { trabalho } \\
\text { Educação no trabalho para o envelhecimento saudável do } \\
\text { trabalhador de enfermagem }\end{array}$ \\
\hline
\end{tabular}

Quadro 1 - Publicações LILACS, do período 1981-2008, sobre o trabalho de enfermagem em uma perspectiva marxiana, segundo elementos articuladores de sua análise, temática de estudo abordada e aspecto enfatizado, Brasil, 2008. 
como no tema anterior, algumas dessas publicações também destacam a dimensão dos sujeitos e/ou de suas interações.

O terceiro tema mais tratado, focando tecnologias do trabalho de enfermagem, ocupa-se de suas bases conceituais, teorizando sobre: os conceitos trabalho e processo de trabaIho, relacionados à enfermagem; as dimensões do trabalho de enfermagem (assistir, gerenciar, educar e pesquisar); o cuidar/ cuidado e/ou o gerenciamento do cuidado de enfermagem; trabalho interdisciplinar na enfermagem; a identidade profissional do enfermeiro; bases orientadoras da assistência e gerenciamento em enfermagem; humanização, comunicação e ética/bioética no trabalho de enfermagem; e a produção social de conhecimentos na enfermagem.

O quarto tema articula processos sociais e/ou institucionais à organização e sujeitos do trabalho de enfermagem, versando sobre: mudanças contemporâneas no mundo do trabalho e suas implicações para a enfermagem e especificamente o cuidar/cuidado em enfermagem; raízes sócio-históricas da prática de enfermagem; conhecimento do trabalhador de enfermagem sobre determinantes e condicionantes de seu trabaIho; divisão técnico-social do trabalho na enfermagem; e condições institucionais relacionadas à prática de enfermagem.

O tema recursos humanos em enfermagem, destacando os trabalhadores como força de trabalho, aborda: a formação/desenvolvimento do trabalhador de enfermagem de forma relacionada a demandas e desafios do trabalho ou ao núcleo cuidar/cuidado em enfermagem; força e mercado de trabalho em enfermagem; e regulação do trabalho de enfermagem. Com maior ênfase nos sujeitos do trabalho de enfermagem, realiza-se o debate da relação trabalhador de enfermagem-trabalho, menos frequente, que trata da produção de subjetividade do trabalhador em questão na prática e da sua educação para o envelhecimento saudável.

O desdobramento do que é enfatizado em períodos (Tabela 1) permite identificar, entre outras, duas características relevantes: 1) ampliação quantitativa de artigos que discutem os sujeitos, suas percepções e/ou interações no trabalho, com concentração nos anos 2000; 2) e inclusão, nesses anos, do debate sobre o cuidado/gerenciamento do cuidado de enfermagem (5 artigos) e a formação/desenvolvimento do trabalhador (5 artigos). Em 1990, 2 publicações focam os sujeitos na prática de enfermagem (ao tratar dos seus elementos constitutivos e da educação para o envelhecimento saudável de seus trabalhadores) e, em 2000, 15 (ao discutir humanização, ética/bioética, comunicação, autonomia profissional, produção da subjetividade dos trabalhadores e modos de efetivar/pensar o trabalho de enfermagem).

Em relação a aspectos metodológicos, encontrou-se que 63 $(64,95 \%)$ artigos são resultados de pesquisas; e 34 (35,05\%) constituem reflexões teóricas. Do total, 91 (93,82\%) publicações adotam uma abordagem qualitativa, 5 (5,15\%) quanti-qualitativa, e 1 (1,03\%) quantitativa. Nos anos 1980, os $2(100 \%)$ artigos encontrados são reflexões teóricas. Nos anos 1990, 11 $(52,38 \%)$ são pesquisas e $10(48,62 \%)$ reflexões teóricas. Nos anos 2000 , cresce a proporcionalidade relativa das pesquisas, correspondendo a $52(70,27 \%)$, enquanto as reflexões teóricas correspondem a $22(29,73 \%)$ artigos do período.
A discussão do trabalho de enfermagem nos anos 1980 é encaminhada através de uma abordagem baseada nas categorias marxianas trabalho, processo de trabalho e correlatos, presente nas $2(100 \%)$ publicações encontradas, referidas à dimensão mais estrutural do trabalho. Nos anos 1990, para além dessas categorias, são incorporados conceitos de outras vertentes teóricas, encontrados em 3 (14,28\%) dos estudos do período. Nos anos 2000, essa característica é mais evidente, encontrada em 25 (33,78\%) artigos.

Destaca-se de forma mais marcante o uso de ideias e conceitos: da análise institucional, com base em Gregorio Franklin Baremblitt, Pierre-Félix Guatarri e Suely Rolnik e/ou Emerson Elias Merhy que neles se pauta; da abordagem filosófica de Michel Foucault sobre saber, poder e controle do corpo; da vertente da psicodinâmica do trabalho ancorada em Christophe Dejours e outros estudiosos; e da teoria da ação comunicativa habermasiana, de Jürgen Habermas. Também são incorporados ao debate em questão ideias e conceitos de intelectuais como Agnes Heller, Pierre Bourdieu, Georges Canguilhem, Hannah Arendt, Antônio Gramsci e Peter Berger e Thomas Luckmann, dentre outros. Encontra-se, ainda, a interface das categorias em questão com a abordagem de gênero na enfermagem.

\section{DISCUSSÃO}

A literatura nacional sobre o trabalho de enfermagem com base em conceitos marxianos e de outras correntes teóricas articuladas caracteriza-se, de forma geral, por sua emergência nos anos 1980 e crescimento subsequente substancial, em especial em anos recentes (2005, 2006 e 2007).

A incorporação de conceitos marxianos nos estudos da prática de enfermagem e o seu crescimento são devedores das relevantes contribuições de pesquisadoras da enfermagem da década de 1980: Maria Cecília Puntel de Almeida, Raimunda Medeiros Germano, Graciette Borges da Silva, Delvair de Brito Alves, Cristina Maria Meira Melo, Brigitta Elza Pfeiffer Castellanos, Semiramis Melani Melo Rocha, Nair Fábio da Silva e Denise Pires.

A produção dessas estudiosas e a que a ela se segue, nos anos 1990 e 2000, situa-se no processo mais abrangente de amadurecimento científico da enfermagem brasileira, impulsionado pela formação de um quadro crescente de mestres e doutores e pela consolidação da pós-graduação na área no país ${ }^{(10)}$, sobretudo a partir da criação do doutorado, em 1981, em meio à criação de condições como: ampliação de revistas científicas da área; crescimento e consolidação de grupos/ linhas de pesquisa voltados a estudos do trabalho de enfermagem; políticas de apoio à pesquisa em enfermagem; e participação da Associação Brasileira de Enfermagem no debate da prática e produção de conhecimentos em enfermagem. Isso tudo mediado pelo saber-fazer cotidiano de enfermeiras brasileiras, em diferentes espaços de atuação, comprometidas com o avanço da enfermagem.

Outras características da literatura analisada compreendem: 1) o destaque à busca de compreensão das contradições, dilemas, permanências, transformações e potencialidades da 
Tabela 1 - Distribuição de artigos da base LILACS, do período 1981-2008, sobre o trabalho de enfermagem, segundo o período e a ênfase do estudo, Brasil, 2008.

\begin{tabular}{|c|c|}
\hline Período & Ênfase do estudo \\
\hline \multirow[t]{2}{*}{ 1980-1989 } & Raízes sócio-históricas da prática de enfermagem/do enfermeiro \\
\hline & Total \\
\hline \multirow[t]{11}{*}{ 1990-1999 } & Modos de efetivar/ pensar o trabalho de enfermagem/do enfermeiro \\
\hline & Elementos constitutivos do trabalho de enfermagem/do enfermeiro \\
\hline & Elementos constitutivos do trabalho de enfermagem/do enfermeiro (destaque às inter-relações/subjetividade) \\
\hline & Força e mercado de trabalho \\
\hline & Mudanças contemporâneas no mundo do trabalho e implicações para a enfermagem \\
\hline & Trabalho de enfermagem/do enfermeiro no trabalho coletivo \\
\hline & Raízes sócio-históricas da prática de enfermagem/do enfermeiro \\
\hline & Categoria trabalho/processo de trabalho como fundamento na enfermagem \\
\hline & Educação para o envelhecimento saudável do trabalhador de enfermagem \\
\hline & Conhecimentos produzidos em enfermagem \\
\hline & Bases orientadoras da prática assistencial e gerencial de enfermagem \\
\hline
\end{tabular}

Modos de efetivar/ pensar o trabalho de enfermagem/do enfermeiro

Total

$\%$

100

$6 \quad 28,57$

29,52

14,76

14,76

Modos de efetivar/ pensar o trabalho de enfermagem/do enfermeiro (destaque às inter-relações/subjetividade)

Elementos constitutivos do trabalho de enfermagem/do enfermeiro

Formação/desenvolvimento do trabalhador e demandas da prática

Formação/desenvolvimento do trabalhador e o cuidar em enfermagem

Elementos constitutivos do trabalho de enfermagem/ enfermeiro (destaque às inter-relações/subjetividade)

Força e mercado de trabalho

Mudanças contemporâneas no mundo do trabalho e implicações para a enfermagem

Mudanças contemporâneas no mundo do trabalho e implicações para o cuidar/cuidado

Trabalho de enfermagem/do enfermeiro no trabalho coletivo

Articulação/ dicotomia entre cuidar e gerenciar no trabalho do enfermeiro

Categoria trabalho/processo de trabalho como fundamento na enfermagem

Conhecimento do trabalhador de enfermagem sobre determinantes e condicionantes de seu trabalho

Cuidado/gerenciamento do cuidado de enfermagem

Trabalho interdisciplinar

Raízes sócio-históricas da prática de enfermagem/do enfermeiro

Autonomia profissional do enfermeiro

Condições institucionais e o trabalho de enfermagem

Dimensões do processo de trabalho de enfermagem

Divisão técnico-social do trabalho em saúde e a enfermagem

Enfermagem como vocação e como trabalho

Enfermagem frente à normatividade institucional

Produção de subjetividade do trabalhador de enfermagem no trabalho

Regulação do trabalho de enfermagem/do enfermeiro

Comunicação no trabalho de enfermagem

Ética/bioética no trabalho gerencial de enfermagem

Trabalho e identidade profissional do enfermeiro

Bases orientadoras da prática assistencial e gerencial de enfermagem

Humanização do trabalho de enfermagem

\section{1}

100

19

25,68

8,11

6,76

4,05

2,70

5,41

5,41

2,70

1,35

4,05

2,70

2,70

2,70

2,70

2,70

1,35

1,35

1,35

1,35

1,35

1,35

1,35

1,35

1,35

1,35

1,35

1,35

2,70

1,35

Total 
prática de enfermagem; 2) crescente ênfase na abordagem dos sujeitos, sobretudo nos anos 2000; 3) e, nessa mesma década, a teorização específica sobre o cuidado de enfermagem e o seu gerenciamento.

Essas características encontram explicação em pelo menos três processos inter-relacionados: 1) na incorporação das ciências sociais no debate científico da saúde e enfermagem e em seus movimentos; 2) no conjunto de mudanças e novas proposições no setor saúde e na participação de sujeitos em torno da reconstrução cotidiana do modelo assistencial predominante; 3) e em características sócio-históricas da prática de enfermagem.

A inclusão das ciências sociais na saúde, no Brasil, ocorre na década de 1970/80, vinculada à constituição do campo da saúde coletiva, abrindo espaço ao recorte de objetos originais de estudo e à interpretação social e política do trabalho em saúde/enfermagem.

Primeiramente, os estudos da saúde coletiva avançam na compreensão da determinação sócio-histórica das práticas em saúde articulando um movimento temático mais geral na produção científica, que resulta em estudos sobre políticas de saúde, organização do setor, processos de trabalho e recursos humanos. É nesse movimento que se insere a produção sobre o trabalho em saúde e enfermagem fundado nas categorias marxianas em questão $\mathrm{O}^{(1,11,12)}$. Em um segundo momento, nos anos 1990, as ciências sociais na saúde travam um debate em torno da crise paradigmática dos modelos teóricos globalizantes, valorizando o estudo de temas microssociais do trabalho e explicações que buscam a mediação dialética entre estrutura e ação social| ${ }^{(11,13)}$.

Na enfermagem, a ênfase nos aspectos microssociais da prática é observada em especial a partir dos anos $1990^{(1)} \mathrm{e}$ relaciona-se, como apontado acima, às mudanças em curso à época e ao núcleo histórico-social de seu trabalho - o cuidado de enfermagem.

No Brasil, sobretudo a partir da segunda metade dos anos 1980, avançam proposições para mudanças no setor saúde, valorizando-se os microespaços de atuação e temas como humanização, comunicação, ética, autonomia/participação, dentre outros, relacionados ao modo de inserção dos trabaIhadores na prática. Sobretudo a partir dos anos 1990, o debate científico, preocupado com permanências e mudanças nas práticas em saúde, repensa os processos de trabalho e a participação cotidiana dos trabalhadores e usuários, tendo em perspectiva a construção de uma atenção mais inclusiva e integral. Assim, a produção encontrada sobre reprodução e inovações do trabalho de enfermagem, além de congregar aspectos históricos da enfermagem, incorpora essa perspectiva em curso, ocupando-se do debate dos modos de efetivar/ pensar a prática de enfermagem por referência a proposições, questões em debate e/ou experimentações no cenário do SUS (como acolhimento, humanização, vigilância à saúde, assistência psicossocial, Programa Saúde da Família, incorporação de tecnologias leves, e outras).

É parte desse movimento, nos anos 2000, a produção sobre a formação e desenvolvimento do trabalhador de enfermagem e o cuidado/gerenciamento do cuidado de enfermagem. No contexto de valorização da atenção integral, o tema cuidado ganha espaço e, também, a necessidade de desenvolver novas competências profissionais através da educação. A específica teorização sobre o cuidado e seu gerenciamento se associa ao seu significado para a enfermagem, na medida em que se relaciona ao núcleo central e específico de seu saber-fazer e ao reconhecimento social e profissional da dupla dimensão de seu trabalho - cuidado e gerenciamento -, problematizada desde os anos 1980.

Em relação aos aspectos metodológicos da produção sobre o trabalho de enfermagem identificou-se: 1) predominância de publicações resultantes de pesquisas qualitativas, a partir dos anos 1990; 2) e introdução de categorias teóricas não marxianas.

A ampliação da literatura derivada de pesquisas é um fenômeno que se associa ao crescimento e consolidação da pós-graduação em enfermagem e ao amadurecimento científico da área a partir dos anos 1980. A predominância dos estudos qualitativos reflete uma tendência mais geral da prática científica brasileira no campo da saúde, em particular da saúde coletiva, do final da década de 1980 e anos 1990, reflexo da crítica aos paradigmas positivistas e da incorporação de novos paradigmas voltados à compreensão social dos fenômenos saúde-doença e do sistema de produção dos serviços de saúde. A tendência à incorporação crescente de estudos qualitativos na enfermagem também é evidenciada na literatura internacional em enfermagem ${ }^{(14)}$.

A introdução de outras categorias na literatura sobre o trabalho de enfermagem se associa ao mencionado movimento disciplinar das ciências sociais em saúde que evidencia limites, possibilidades e mudanças dos paradigmas que norteiam a ciência contemporânea ${ }^{(13)}$. Como parte da abertura interdisciplinar em saúde, na segunda metade dos anos 1990, já se identifica na produção da pós-graduação da enfermagem a inclusão de conceitos de outras abordagens teóricas no debate da objetividade-subjetividade do trabalho ${ }^{(1)}$.

Nos anos 1990, Mendes-Gonçalves e outros estudiosos da saúde coletiva que adotam conceitos marxianos do trabalho dialogam com intelectuais de outras filiações, como, Agnes Heller, José Arthur Gianotti, Theodor Adorno, Jürgen Habermas, Gaston Bachelard e Georges Canguilhem, destacando aspectos dinâmicos e relacionais do trabalho em saúde necessários para pensá-lo além de sua dimensão estrutural ${ }^{(3)}$. Emerson Merhy, em especial, influi de forma marcante os estudos da enfermagem ao articular conceitos marxianos e de analistas institucionais, revalorizando a dimensão da subjetividade na compreensão das práticas. Na enfermagem, nesses anos, autores como Silvana Mishima, Marina Peduzzi, Flávia Regina Ramos, entre outros, vão demarcar a incorporação dessa nova perspectiva epistemológica nos estudos da prática e influir na trajetória acadêmica das produções sobre o trabalho de enfermagem nos anos 2000.

A articulação de categorias marxianas com outras correntes teóricas reflete, além de movimentos em prol da ampliação dos horizontes da ciência em enfermagem, o reconhecimento dos limites do quadro teórico do trabalho em saúde e enfermagem para a análise das mudanças na concepção do 
processo saúde-doença e no processo de trabalho, em especial para a discussão do cuidado que é processo demarcado pela participação de sujeitos em interação social.

\section{CONSIDERAÇÕES FINAIS}

A literatura crescente sobre o trabalho de enfermagem à luz de categorias marxianas e, mais recentemente, também de outras correntes teóricas, vem contribuindo à compreensão de como a enfermagem está inserida no campo da saúde, como executa seu trabalho cotidiano e seus significados para a própria profissão e para os sujeitos envolvidos, propiciando a produção de um conhecimento próprio da profissão e certo destaque a ação dos sujeitos em sua configuração.
$\mathrm{Na}$ literatura produzida destaca-se o debate sobre o modo como a Enfermagem/enfermeiro participa da reprodução-transformação das práticas hegemônicas, bem como o espaço social e de poder que ocupa no trabalho coletivo em saúde, reconhecidamente minoritário no sentido do valor social que lhe é atribuído. Dada a frágil visibilidade do trabalho de enfermagem e sua heterogeneidade, a produção teórica coloca no centro do debate aspectos relacionados ao que somos, ao que fazemos, e para onde vamos enquanto prática social. Observa-se, nas publicações, a busca pela compreensão das contradições e potenciais cujo esclarecimento fundamentado permita configurar uma prática de enfermagem com mais amplo reconhecimento social de seus agentes e maior impacto na qualidade dos cuidados de enfermagem de usuários e população.

\section{REFERÊNCIAS}

1. Almeida MCP, Mishima SM, Peduzzi M. A pesquisa em enfermagem fundamentada no processo de trabalho: em busca da compreensão e qualificação da prática de enfermagem. $51^{\circ}$ Congresso Brasileiro de Enfermagem, $10^{\circ}$ Congresso Panamericano de Enfermeria; 1999 out 2-7; Florianópolis, Brasil. Florianópolis: ABEn; 1999. p. 259-77.

2. Almeida MCP, Silva GB, Ruffino MC, Steagall-Gomes DI, Yazlle-Rocha JS, Germano RM. Contribuições ao estudo da prática de enfermagem - Brasil [Mimeografado]; 1981.

3. Ayres JRCM. Teoria do trabalho e construção de conhecimento em saúde coletiva: para uma epistemologia reconstruída como práxis emancipadora. In: Do processo de trabalho em saúde à dialógica do cuidado: repensando conceitos e práticas em saúde coletiva [livre-docência]. São Paulo: Departamento de Medicina Preventiva/USP; 2002. p. 13-20.

4. Marx K. O capital. 14ed. São Paulo: Difel; 1998. v.1.

5. Mendes-Gonçalves RB. Práticas de saúde: processo de trabalho e necessidades. Cadernos CEFOR, São Paulo: Secretaria Municipal de Saúde; 1992.

6. Fracolli LA, Granja GF. A utilização da categoria processo de trabalho pela enfermagem brasileira: uma análise bibliográfica. Rev Esc Enferm USP 2005;39(n. esp.):597-602.

7. Barbosa LR, Melo MRAC. Relações entre qualidade da assistência de enfermagem: revisão integrativa da literatura. Rev Bras Enferm 2008;61(2):366-70.

8. Ministério da Ciência e Tecnologia (BR). Conselho Nacional de Desenvolvimento Científico e Tecnológico. Diretório dos Grupos de Pesquisa no Brasil. [Acesso em 2008 Ago 20]. Disponível em: http://dgp.cnpq.br/buscaoperacional/.

9. Brasil. Biblioteca Virtual em Saúde. Portal de Revistas Científicas em Ciências da Saúde [Acesso em 2008 Ago 20]. Disponível em: http://portal.revistas.bvs.br/

10. Erdmann AL, Fernandes JD. Publicações científicas qualificadas na enfermagem brasileira. Rev Bras Enferm 2009;62(4):499.

11. Nunes ED. A trajetória das ciências sociais em saúde na América Latina: revisão da produção científica. Rev Saúde Públ. 2006;40(n.esp.):64-72.

12. Trezza MCA. Figueiredo RMS, Leite JL. Enfermagem como prática social: um exercício de reflexão. Rev Bras Enferm 2008;61(6):904-8.

13. Burlandy L, Bodstein RC. Política e saúde coletiva: reflexão sobre a produção científica (1976-1992). Cad Saúde Públ. 1998;14(3):543-54.

14. Silva LMS, Oliveira NRN, Frota MA, Fialho AVM. Pesquisa internacional em enfermagem: tendências temáticas e metodológicas. Rev Bras Enferm 2008;61(5):615-9. 